\title{
PRIORIZAÇÃO DE PROJETOS DE MELHORIA DE PRODUTIVIDADE ATRAVÉS DO MÉTODO MULTICRITÉRIO PROMÉTHÉE II ${ }^{1}$
}

\author{
Bruno Carlos da Silva Sousa ${ }^{\text {a*}}$, Luís Alberto Duncan Rangel ${ }^{\mathrm{a}}$, Cecilia Toledo Hernández ${ }^{\mathrm{a}}$

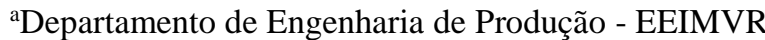 \\ Universidade Federal Fluminense - UFF, Volta Redonda-RJ, Brasil
}

Recebido 20/10/2017, aceito 13/03/2018

\begin{abstract}
RESUMO
O processo de elaboração do portfólio de projetos é uma tarefa de difícil de decisão, sobretudo no âmbito da produtividade. As empresas buscam se tornar mais produtivas e competitivas com a realização de projetos que apresentam diferentes níveis de retornos financeiros, esforços, prazos e probabilidade de sucesso. A tomada de decisão nas organizações ocorre na presença de múltiplos critérios, quantitativos e qualitativos, frequentemente conflitantes, e torna-se indispensável o emprego de métodos multicritério. Assim, este artigo apresenta a aplicação do método multicritério PROMÉTHÉE II na priorização de projetos de produtividade em uma empresa de grande porte, servindo de suporte no planejamento do portfólio anual de projetos. Os resultados mostram que a aplicação de métodos multicritério torna mais clara a solução de conflitos existentes entre os critérios e permite aumentar a racionalidade presente no processo de decisão, auxiliando na definição de prioridades.
\end{abstract}

Palavras-chave: Priorização de projetos; Apoio multicritério à decisão; PROMÉTHÉE II

\begin{abstract}
The process of developing project portfolios is a difficult decision-making task, especially in the area of productivity. Companies seek to become more productive and competitive by carrying out projects which present different levels of financial returns, efforts, deadlines and probability of success. In organizations, decision making are based on multiple quantitative and qualitative criteria, often conflicting. Thus, it becomes indispensable to use multicriteria methods. In this regard, this article presents the application of the multicriteria method PROMÉTHEE II to the prioritization of productivity projects in a large company, serving as support in the planning of the annual project portfolio. The results show that the application of multicriteria methods helps to solve existing conflicts among the criteria and it allows the increase of rationality present in the decision processes by helping the definition of priorities.
\end{abstract}

Keywords: Prioritization of projects; Multicriteria decision aid; PROMÉTHÉE II.

*Autor para correspondência. E-mail: brunocarloss@ hotmail.com

DOI: 10.4322/PODes.2018.003 


\section{Introdução}

Em um mercado cada vez mais competitivo, as empresas buscam oferecer produtos e serviços que maximizem a satisfação dos seus clientes, com objetivo de fornecê-los na mais alta qualidade sob o desafio constante do melhor preço. Segundo Dennis (2008), o grande desafio do século 21 é a redução de custo. $\mathrm{O}$ acesso sem precedentes à informação, a concorrência globalizada e a vasta variedade de escolhas, favorecem o aumento da exigência dos consumidores por excelente qualidade a um preço razoável. Neste sentido, as organizações devem continuamente melhorar o desempenho de seu sistema de produção em prol da redução de seus custos de fabricação, e um de seus grandes componentes é a baixa produtividade industrial (Andersson e Bellgran, 2015).

Ser competitivo é oferecer produtos com maior qualidade, gerar satisfação no cliente e alcançar custos que permitam rentabilidade compatível com o setor de atuação. Neste sentido, as organizações baseiam-se numa eficiente utilização de recursos disponíveis para a produção, tanto operacionais quanto humanos (Mercado e Cleto, 2012).

Os projetos de produtividade são alavancas para o aumento de desempenho das empresas. King et al. (2014) apontam que os indicadores de produtividade foram inseridos nas empresas para que o desempenho organizacional pudesse ser medido, analisado e otimizado. O processo de escolha e definição destes projetos tem como objetivo buscar a maximização dos ganhos líquidos na sua realização. Porém, estes apresentam diferentes níveis de retornos financeiros, esforços, prazos e probabilidade de sucesso, além de sempre considerar as restrições de recursos humanos para realização.

A priorização de portfólio de projetos se enquadra como um problema complexo. O problema se caracteriza, segundo Macedo (2008), pela disposição de um agente de decisão de escolher uma ação entre diversas possibilidades, denominadas de alternativas, de maneira a selecionar a considerada mais satisfatória. Ele também se caracteriza pela existência de múltiplos critérios, às vezes conflitantes. Ao tomar decisões sobre portfólio, deve-se considerar o trade-off intrínseco, pois más decisões implicam em altos custos de oportunidade (Kleinmuntz, 2007).

Segundo Gomes (2016), uma vez que a tomada de decisão nas organizações normalmente ocorre na presença de múltiplos critérios, quantitativos e qualitativos, torna-se indispensável o emprego de métodos de apoio multicritério à decisão (AMD). A aplicação destes métodos fornece um suporte adequado aos decisores, devido à capacidade de elevar a racionalidade do processo e busca amenizar eventual viés causado por decisões intuitivas e/ou influências políticas (Montibeller e Franco, 2007).

Diversos problemas de AMD fazem uso do PROMÉTHÉE II na priorização de projetos, como podem ser vistos em (Pereira, 2016; Shakasi-Niaei et al., 2011; Bin et al., 2015). Estas pesquisas mostram a eficácia na utilização deste método em diversas áreas e setores industriais, podendo ser também de grande contribuição no âmbito da melhoria de produtividade.

Neste contexto, esta pesquisa tem como objetivo a aplicação do método PROMÉTHÉE II na priorização de projetos voltados à melhoria de produtividade dentro de um portfólio de projetos de uma indústria, evidenciando os principais critérios utilizados neste processo de avaliação. Inicialmente, serão abordados os principais conceitos do termo Produtividade e Projetos, seguido da apresentação das fundamentações teóricas do método PROMÉTHÉE II. Finalmente serão exibidos os resultados da aplicação do AMD na priorização de projetos em um Estudo de Caso de uma empresa multinacional de grande porte.

\section{Produtividade}

Produtividade é um indicador de desempenho chave em todos os níveis, desde o processo de produção de uma empresa até a economia nacional (Demeter et al., 2011). O conceito, quando aplicado a um sistema físico de produção, é definido como a relação entre o que é obtido na saída e o que é consumido na entrada desse sistema (Sink, 1985). 
A produtividade nas indústrias também pode ser entendida como a eficiência com a qual os insumos são transformados em produção. Nesse caso, a medição da produtividade é feita principalmente por indicadores de natureza físico-operacional, como produção realizada por unidade de tempo (Macedo, 2012).

Segundo a Confederação Nacional das Indústrias (CNI, 2015), a produtividade no Brasil cresceu bem menos quando comparada a outros países, no período entre 2002 e 2012. Este indicador colabora para o crescimento do custo unitário do trabalho, que sofreu um grande aumento na década passada. Vegunta e Milanovic (2011) afirmam que a competitividade no mercado no qual as empresas estão inseridas faz com que a eficiência operacional seja tratada como prioridade de negócio. O desafio e a questão chave consistem em encontrar como transformar entradas disponíveis de um processo em saídas desejadas da forma mais eficiente e eficaz possível (Käpylä et al., 2010).

Severiano Filho (1998) toma como referência três definições do termo produtividade: i) Produtividade de Fator Simples, referente à relação de produção com apenas um dos insumos usados no processo produtivo; ii) Produtividade de Valor Agregado, referente à relação entre o valor agregado e os diversos recursos de produção utilizados e iii) Produtividade de Fator Total, considerando simultaneamente mais de um insumo nos recursos.

Este trabalho utiliza o conceito clássico de produtividade do fator simples. Os projetos voltados à melhoria de produtividade são aqueles destinados ao aumento da capacidade produtiva e/ou redução dos recursos humanos necessários no processo.

\subsection{Projetos de Produtividade}

Entendem-se como projeto, as atividades sistematicamente ordenadas que sejam realizadas em sequência e que tenham início, meio e fim determinados fornecendo um resultado único. Para Kerzner (2006), projeto é um empreendimento com objetivo bem definido, que consome recursos e opera sob pressões de prazos, custos e qualidade. Este conceito pode ser resumido na afirmação de que um projeto consiste em um esforço temporário empreendido para criar um produto, serviço ou resultado exclusivo (PMI, 2013).

Estes aspectos são reforçados nas palavras de Keelling (2006), que diz que um projeto é planejado, financiado e administrado como uma atividade distinta e estando divorciado do trabalho de rotina, é mais fácil de ser planejado, monitorado e controlado.

Quando se trata de um conjunto de projetos realizados, administrados e controlados de maneira coordenada tem-se, então, um portfólio. Os portfólios são agrupados para facilitar o gerenciamento eficaz desse trabalho a fim de atender aos objetivos de negócios estratégicos (PMI, 2013). Almeida e Duarte (2011) definem portfólio como o conjunto de projetos a serem implementados que busquem satisfazer objetivos da organização sem consumir mais recursos do que os disponíveis nem violar qualquer outra restrição imposta pelo problema.

$\mathrm{O}$ efeito gerado pelas orientações estratégicas das organizações sobre o sucesso do negócio é medido pela estruturação e sucesso do portfólio de projetos (Meskendahl, 2010). As atividades típicas no escopo deste portfólio são a coleta de possíveis projetos, sua priorização e seleção de acordo com os recursos disponíveis e a avaliação de projetos em execução (Kaiser et al., 2015).

O portfólio de projetos de produtividade trata-se de um conjunto de projetos estruturados objetivando ganhos no âmbito da produtividade. Estes projetos são realizados voltados a otimização dos processos industriais, através do aumento da produção e minimização dos recursos trabalhados.

\section{Apoio Multicritério à Decisão}

Uma organização frequentemente se encontra diante de problemas sérios de decisão (Shimizu, 2006). Entende-se como decisão, o processo no qual uma escolha de alternativa é realizada dentro de várias alternativas (Belton e Stewart, 2002). Decidir é uma ação humana, 
quando se refere à necessária racionalidade na escolha de alguma entre as alternativas que surjam e que sejam apresentadas na rotina diária da empresa (Rossoni, 2011).

O Apoio Multicritério à Decisão (AMD) é uma ferramenta para apoiar à tomada de decisão e consolidou-se com um enfoque adequado ao tratamento de problemas envolvendo escolha, classificação ou priorização de alternativas na presença de critérios múltiplos e conflitantes (Gomes, 2016). Ele consiste em escolher a melhor alternativa dentro de um conjunto de duas ou mais alternativas, baseando esta decisão em dois ou mais critérios (Almeida, 2013). Esta metodologia pode considerar em suas aplicações o fator humano e subjetividade, inerente ao problema de decisão (Gomes et al., 2009).

Existem diversos tipos de métodos multicritério, entre os quais, destacam-se os Compensatórios e os de Sobreclassificação. Os métodos compensatórios, em destaque o AHP (Saaty, 1980), agregam os critérios em um único critério de síntese. Estes métodos pressupõem a condição do decisor de obter uma concepção exata sobre a utilidade dos scores de cada alternativa e dos pesos de cada critério, com uma metodologia baseada em análise hierárquica dos dados (Ferreira, 2013)

Já os métodos de sobreclassificação, destacando o ELECTRE (Roy, 1968) e PROMÉTHÉE (Brans et al., 1984), são baseados em dominância, ou seja, a busca por um grupo de alternativas cujo desempenho é melhor ou igual as demais alternativas em todos os critérios (Almeida, 2013). Eles empregam a relação de sobreclassificação, realizando comparação par a par das alternativas presentes no processo decisório.

Uma boa referência teórica sobre os métodos ELECTRE e PROMETHÉE podem ser vistos em (Govindan e Jepsen, 2016; Behzadian et al., 2010), respectivamente.

\subsection{Método PROMÉTHÉE II}

O método PROMÉTHÉE (Preference Ranking Organization Method for Enrichment Evaluations) trata-se de uma variação do método ELECTRE, com uma maior resistência às variações dos parâmetros, mas sujeitos às subjetividades dos parâmetros técnicos (Campos, 2011). É considerado umas das principais metodologias de avaliação multicritério por sua facilidade de aplicação e entendimento.

Este método utiliza uma relação de dominância, onde se busca encontrar uma relação de superação que leva em conta o conjunto dos critérios propostos. Para cada par de alternativas se estabelece um grau geral de preferência de uma sobre a outra (Macedo, 2008). As diversas variações do PROMÉTHÉE descritas na literatura (Brans e Mareschal, 2005) são as seguintes:

- PROMÉTHÉE I (Brans et al., 1984) - utilizada em problemática de escolha, estabelecendo uma pré-ordem parcial entre as alternativas.

- PROMÉTHÉE II (Brans et al., 1984) - aplicada em problemática de ordenação, através de pré-ordem completa entre as alternativas.

- PROMÉTHÉE III (Brans et al., 1986) - ampliada a noção de indiferença, com tratamento probabilístico dos fluxos.

- PROMÉTHÉE IV (Brans et al., 1986) - utilizada em problemáticas de escolha e ordenação, em situações com conjunto de soluções viáveis contínuo.

- PROMÉTHÉE V (Brans e Mareschal, 1992) - implementada em problemáticas com necessidade de priorização de alternativas, sujeitas a restrições. Uma lógica de programação inteira é estabelecida.

- PROMÉTHÉE VI (Brans e Mareschal, 1995) - destinada às situações em que o decisor não consegue estabelecer um valor fixo de peso para cada critério.

- PROMÉTHÉE GAIA (Brans e Mareschal, 1994) - extensão dos resultados do PROMÉTHÉE, através de um procedimento visual e interativo.

Segundo Behzadian et al. (2010), o método PROMÉTHÉE II destina-se a fornecer uma classificação completa de um conjunto finito de alternativas da melhor a pior. Para aplicação deste método faz-se necessário definir a matriz de desempenho das alternativas que será a base 
para a aplicação desta metodologia. Esta matriz apresenta o desempenho obtido por cada alternativa em relação a cada critério de avaliação presente na análise. Na Tabela 1 pode-se observar a representação da matriz de desempenho das alternativas.

Tabela 1: Matriz de desempenho das alternativas.

\begin{tabular}{|c|c|c|c|c|c|}
\hline \multirow{2}{*}{ Alternativa } & \multicolumn{5}{|c|}{ Critérios } \\
\cline { 2 - 6 } & $\mathrm{C}_{1}$ & $\mathrm{C}_{2}$ & $\mathrm{C}_{3}$ & $\ldots$ & $\mathrm{C}_{\mathrm{m}}$ \\
\hline $\mathrm{A}_{1}$ & $\mathrm{~g}_{1}\left(\mathrm{~A}_{1}\right)$ & $\mathrm{g}_{2}\left(\mathrm{~A}_{1}\right)$ & $\mathrm{g}_{3}\left(\mathrm{~A}_{1}\right)$ & $\ldots$ & $\mathrm{g}_{\mathrm{m}}\left(\mathrm{A}_{1}\right)$ \\
\hline $\mathrm{A}_{2}$ & $\mathrm{~g}_{1}\left(\mathrm{~A}_{2}\right)$ & $\mathrm{g}_{2}\left(\mathrm{~A}_{2}\right)$ & $\mathrm{g}_{3}\left(\mathrm{~A}_{2}\right)$ & $\ldots$ & $\mathrm{g}_{\mathrm{m}}\left(\mathrm{A}_{2}\right)$ \\
\hline $\mathrm{A}_{3}$ & $\mathrm{~g}_{1}\left(\mathrm{~A}_{3}\right)$ & $\mathrm{g}_{2}\left(\mathrm{~A}_{3}\right)$ & $\mathrm{g}_{3}\left(\mathrm{~A}_{3}\right)$ & $\ldots$ & $\mathrm{g}_{\mathrm{m}}\left(\mathrm{A}_{3}\right)$ \\
\hline$\ldots$ & $\ldots$ & $\ldots$ & $\ldots$ & $\ldots$ & $\ldots$ \\
\hline $\mathrm{A}_{\mathrm{n}}$ & $\mathrm{g}_{1}\left(\mathrm{~A}_{\mathrm{n}}\right)$ & $\mathrm{g}_{2}\left(\mathrm{~A}_{\mathrm{n}}\right)$ & $\mathrm{g}_{3}\left(\mathrm{~A}_{\mathrm{n}}\right)$ & $\ldots$ & $\mathrm{g}_{\mathrm{m}}\left(\mathrm{A}_{\mathrm{n}}\right)$ \\
\hline
\end{tabular}

Fonte: Brans et al. (1984).

O decisor deve estar esclarecido acerca dos critérios, de forma que esteja apto a traduzir em números a importância relativa destes critérios de acordo com as preferências do decisor (Almeida, 2012), como mostra a Tabela 2. Esta importância relativa $\left(\mathrm{W}_{\mathrm{j}}\right.$ ) é a constante de escala do critério j avaliado pelo PROMÉTHÉE II, e o somatório dos pesos deve atender as condições da Equação 1.

Tabela 2: Constante de escala dos critérios.

\begin{tabular}{|c|c|c|c|c|c|}
\hline Critérios & $\mathrm{C}_{1}$ & $\mathrm{C}_{2}$ & $\mathrm{C}_{3}$ & $\ldots$ & $\mathrm{C}_{\mathrm{m}}$ \\
\hline Constante Escala & $\mathrm{w}_{1}$ & $\mathrm{w}_{2}$ & $\mathrm{w}_{3}$ & $\ldots$ & $\mathrm{w}_{\mathrm{m}}$ \\
\hline
\end{tabular}

Fonte: Brans et al. (1984).

$$
\sum_{j=1}^{n} w_{j}=1
$$

Os desempenhos das alternativas em relação aos critérios, no método PROMÉTHÉE II, são verificados através de seis funções de preferências (Brans et al., 1986). Para cada critério, a função de preferência traduz a diferença entre as avaliações obtidas por duas alternativas em um grau de preferência variando de zero a um (Behzadian et al., 2010). A escolha dessas funções é de responsabilidade dos decisores que buscam representar a forma em que a diferença de desempenho entre duas alternativas é considerada em cada critério de avaliação.

Para algumas dessas funções de preferência é necessário atribuir valores para os limites de preferência (p) e de indiferença (q). O limite (q) representa um limite de indiferença, o maior valor para $\left[F_{j}(a)-F_{j}(b)\right]$ abaixo do qual existe uma indiferença. $O$ limite $(p)$ representa o limite de preferência, o menor valor para $\left[\mathrm{F}_{\mathrm{j}}(\mathrm{a})-\mathrm{F}_{\mathrm{j}}(\mathrm{b})\right]$ acima do qual existe uma preferência estrita (Cavalcante e Almeida, 2005).

Segundo Brans et al. (1986), as funções de preferências utilizadas no método PROMÉTHÉE II podem ser representadas conforme Figura 1.

- Verdadeiro Critério: A função assume valor zero se a diferença de desempenho entre as alternativas for menor ou igual a zero e assume valor 1 se a diferença for maior que zero.

- Quase Critério: A função assume valor zero se a diferença de desempenho entre as alternativas for menor que (q) e assume valor 1 se a diferença for maior ou igual este índice.

- Pseudo Critério: A função permanece em seu ponto de máximo quando a diferença de desempenho for superior ao índice de preferência (p) e será nula para diferenças de desempenho menor que zero. Nesta função existe um crescimento linear da função no intervalo $0 \leq \mathrm{D}_{\mathrm{i}}(\mathrm{a}, \mathrm{b}) \leq \mathrm{p}$. 
- Critério de Nível: A função apresenta valor máximo para diferenças de desempenho superiores a (p) e valor nulo para diferenças de desempenho inferiores a (q). Para o critério de nível $\mathrm{F}_{\mathrm{i}}(\mathrm{a}, \mathrm{b})$ assume valor 0,5 para o intervalo $\mathrm{q}<\mathrm{D}_{\mathrm{i}}(\mathrm{a}, \mathrm{b}) \leq \mathrm{p}$.

- Critério Preferência Linear: A função permanece em seu ponto de máximo quando a diferença de desempenho é superior ao índice de preferência (p) e será nula para diferenças de desempenho menor que o índice de indiferença (q). No critério de preferência linear existe um crescimento da função para o intervalo de $\mathrm{q} \leq \mathrm{D}_{\mathrm{i}}(\mathrm{a}, \mathrm{b}) \leq \mathrm{p}$.

- Critério de Gauss: A preferência cresce seguindo uma curva normal.

Figura 1: Funções de preferência.

\begin{tabular}{|c|c|c|}
\hline \\
\hline 1-Função Verdadeiro Critério \\
\hline
\end{tabular}

Fonte: Brans et al. (1986).

Estabelecidas as intensidades de preferências, obtém-se o grau de sobreclassificação $\pi$ (a,b) para cada par de alternativas (a,b) (Vincke, 1992), sendo calculado conforme Equação 2.

$$
\pi(a, b)=\frac{1}{W} \sum_{i=1}^{m} W_{i} F_{i}(a, b)
$$

Em seguida, calculam-se os fluxos de saída e entrada. Esta forma de sobreclassificação serve para indicar o quanto uma alternativa sobreclassifica as demais (fluxo de saída) e o quanto ela é sobreclassificada (fluxo de entrada), mostrado pelas Equações 3 e 4, respectivamente. O fluxo líquido final é calculado pela diferença entre os dois fluxos, conforme Equação 5.

$$
\begin{aligned}
& \phi^{+}(a)=\frac{1}{n-1} \sum \pi(a, b) \\
& \phi^{-}(a)=\frac{1}{n-1} \sum \pi(b, a) \\
& \phi(a)=\phi^{+}(a)-\phi^{-}(a)
\end{aligned}
$$

O PROMÉTHÉE II estabelece uma ordem decrescente de classificação ordenando todas as alternativas através do fluxo líquido ф que servirá de apoio ao tomador de decisão.

Os métodos da família PROMÉTHÉE podem ser aplicados com resultados satisfatórios em diversas áreas, como apresentados por (Fontana e Morais, 2017) na priorização de válvulas em rede de segmentação; (López e Almeida, 2014) na seleção de projetos estratégicos na área de energia elétrica; (Figueiredo e Silva, 2014) na priorização de portfólio de produtos em 
ambiente agroindustrial. A série de métodos desta família oferece aos tomadores de decisão, a possibilidade de compreender conceitos e parâmetros que simplificam o processo de modelagem de preferências (Silva et al., 2015). A aplicação prática na indústria do AMD requer a utilização de métodos que apresentem clareza e estabilidade, características estas do PROMÉTHÉE.

A utilização dos métodos de sobreclassificação, através da relação de dominância e grau de preferência entre as alternativas, conforme (Macedo, 2008), podem ser de grande importância nos processos de seleção de projetos. Nas empresas, o bom desempenho de determinado projeto em um dado critério não compensa um mau desempenho em outro, e o grau de preferência entre projetos não seriam observados utilizando métodos com critério de síntese.

\section{Estudo de Caso}

A pesquisa realizada destina-se a aplicação de um método multicritério na priorização do portfólio de projetos de melhoria de produtividade. A Figura 2 apresenta as etapas de construção do modelo nesta pesquisa.

Figura 2: Etapas de construção do modelo.

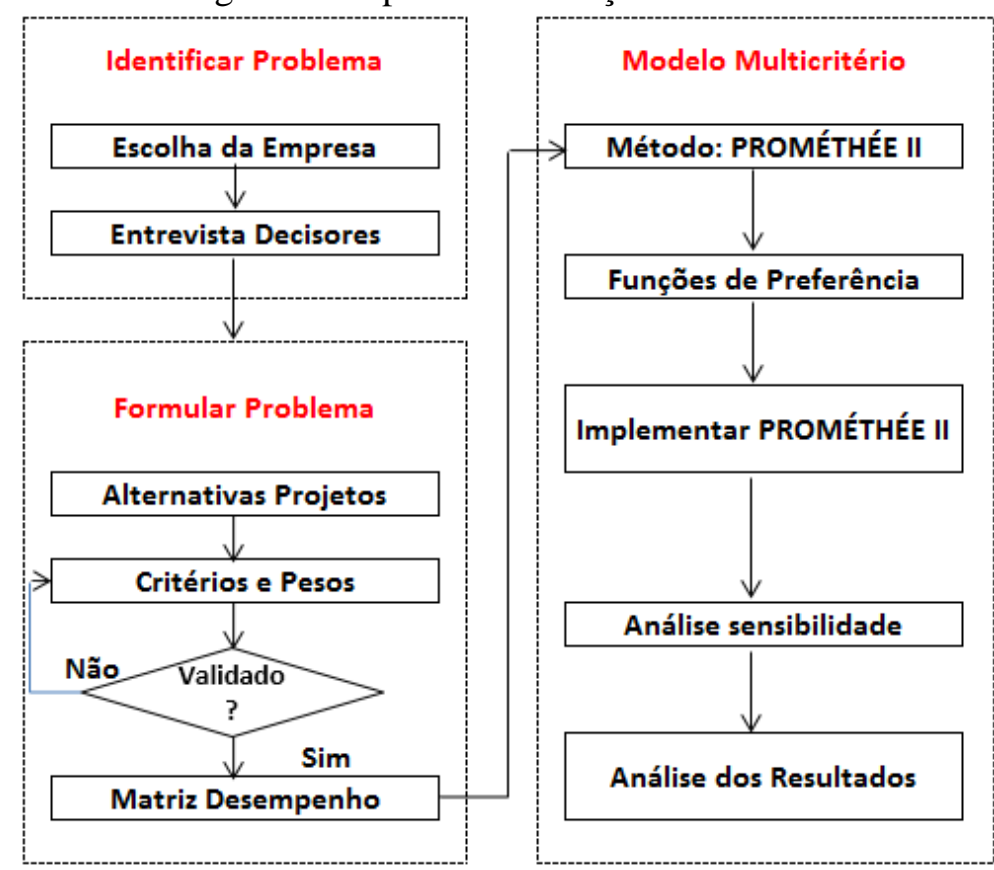

Fonte: Elaborado pelos autores.

O Estudo de Caso é aplicado em uma empresa multinacional de grande porte situada na região sudeste, com o objetivo de priorizar o conjunto de projetos de melhoria de produtividade que a empresa visa executar em um ano. A empresa define quais projetos, destinados a produtividade, irão compor o portfólio anual, muitas vezes de forma subjetiva com os decisores, membros da direção da empresa. A seleção ocorre através de julgamentos de prioridade, dados pelos decisores, e um planejamento é criado com a lista ordenada de projetos a realizar.

Neste artigo, foi escolhido um setor de fabricação desta empresa que possuía 10 projetos inseridos dentro do portfólio de produtividade. Estes projetos compõem as alternativas dentro da metodologia multicritério, avaliadas segundo os critérios definidos. Para tanto, foi elaborada uma entrevista junto a dois decisores, gerentes de fabricação da empresa e responsáveis pelo processo de escolha, para definição dos critérios e pesos associados. Os critérios definidos são os seguintes:

$\mathrm{C}_{1}$ : Ganho Financeiro: Critério quantitativo de maximização que representa o ganho anual com a realização do projeto. As alternativas são avaliadas em milhares de reais por ano. 

meses.

$\mathrm{C}_{2}$ : Prazo: Critério quantitativo de minimização que representa prazo do projeto em

$\mathrm{C}_{3}$ : Atualização de Referência: Critério qualitativo de maximização que mede a contribuição do projeto na coleta de dados de referência (tempo de ciclo, rendimentos, ocupações dos postos, etc.). Nesta avaliação, considera-se 1 para não obtenção de dados de referência, 2 para dados de referência de pouca importância e 3 para dados de referência de alta importância.

$\mathrm{C}_{4}$ : Probabilidade de Sucesso: Critério qualitativo de maximização que mede a expectativa de obtenção dos resultados do projeto, atrelado à sua complexidade. Considera-se 1 para baixa probabilidade de sucesso, 2 para média probabilidade e 3 para alta probabilidade.

$\mathrm{C}_{5}$ : Ganho de Capacidade de Produção: Critério qualitativo de maximização que avalia se o projeto possui ganhos de aumento de capacidade de produção no posto de trabalho. Considera-se 1 para projetos sem aumento de capacidade e 2 para projetos com aumento de capacidade.

Os pesos dos critérios foram obtidos em consenso em uma reunião com os dois decisores e o critério $\mathrm{C}_{5}$ foi definido como o mais importante pelo impacto nos resultados da empresa, frente aos seus objetivos, com constante de escala normalizada igual a 0,33. As constantes de escala dos demais critérios foram definidas através do método direct rating $(100 \%, 80 \%, 50 \%$, $50 \%, 20 \%$ ), respectivamente, para os critérios $\mathrm{C}_{5}, \mathrm{C}_{1}, \mathrm{C}_{2}, \mathrm{C}_{4}, \mathrm{C}_{3}$, conforme Tabela 3.

Tabela 3: Definição das constantes de escala dos critérios.

\begin{tabular}{|c|c|c|c|c|c|}
\hline Critérios & $\mathbf{C}_{\mathbf{1}}$ & $\mathbf{C}_{\mathbf{2}}$ & $\mathbf{C}_{\mathbf{3}}$ & $\mathbf{C}_{\mathbf{4}}$ & $\mathbf{C}_{\mathbf{5}}$ \\
\hline Valores Normalizados & 0,27 & 0,17 & 0,07 & 0,17 & 0,33 \\
\hline
\end{tabular}

Fonte: Elaborado pelos autores.

A matriz de desempenho das alternativas, representada na Tabela 4, foi criada com apoio dos especialistas da empresa para a avaliação dos projetos em cada critério. Para o critério $\mathrm{C}_{1}$, os resultados da matriz foram normalizados para garantir a confidencialidade das informações.

Tabela 4: Matriz de desempenho das alternativas.

\begin{tabular}{|c|c|c|c|c|c|}
\hline \multirow{2}{*}{ Projetos } & \multicolumn{5}{|c|}{ Critérios } \\
\cline { 2 - 6 } & $\mathrm{C}_{1}$ & $\mathrm{C}_{2}$ & $\mathrm{C}_{3}$ & $\mathrm{C}_{4}$ & $\mathrm{C}_{5}$ \\
\hline $\mathrm{A}$ & 1,0 & 3 & 2 & 3 & 2 \\
\hline $\mathrm{B}$ & 0,5 & 2 & 2 & 2 & 1 \\
\hline $\mathrm{C}$ & 0,5 & 2 & 1 & 2 & 2 \\
\hline $\mathrm{D}$ & 0 & 1 & 2 & 3 & 1 \\
\hline $\mathrm{E}$ & 0 & 2 & 2 & 2 & 1 \\
\hline $\mathrm{F}$ & 0 & 3 & 3 & 3 & 1 \\
\hline $\mathrm{G}$ & 0,5 & 6 & 1 & 1 & 1 \\
\hline $\mathrm{H}$ & 1,0 & 4 & 2 & 1 & 2 \\
\hline $\mathrm{I}$ & 0,2 & 2 & 1 & 2 & 1 \\
\hline $\mathrm{J}$ & 0,5 & 3 & 1 & 2 & 1 \\
\hline
\end{tabular}

Fonte: Elaborado pelos autores.

As funções de preferências utilizadas na pesquisa são aquelas que geram uma maior gama de valores intermediários. A escolha dos parâmetros de indiferença q e preferência p para cada critério foi validada junto aos decisores segundo seus julgamentos. A função de preferência de Verdadeiro Critério foi aplicada ao critério $\mathrm{C}_{5}$, pois trata-se de um critério qualitativo sob duas únicas opções de avaliação. Da mesma forma, a função Critério de Nível foi aplicada aos critérios $\mathrm{C}_{3}$ e $\mathrm{C}_{4}$ por se tratar de critérios qualitativos com três opções de avaliação. E a função Pseudo Critério foi aplicada aos critérios $\mathrm{C}_{1}$ e $\mathrm{C}_{2}$, pois nestes critérios quantitativos se torna interessante avaliar o crescimento linear da preferência de uma alternativa pela outra. A Tabela 5 apresenta as funções de preferências e os índices q e p de cada critério. 
Tabela 5: Funções de preferência dos critérios.

\begin{tabular}{|l|c|c|c|}
\hline \multicolumn{1}{|c|}{ Critério } & Função & $\mathbf{q}$ & $\mathbf{p}$ \\
\hline $\mathrm{C}_{1}$ Ganho & III & NA & 150 \\
\hline $\mathrm{C}_{2}$ Prazo & III & NA & 5 \\
\hline $\mathrm{C}_{3}$ Referência & IV & 1 & 2 \\
\hline $\mathrm{C}_{4}$ Sucesso & IV & 1 & 2 \\
\hline $\mathrm{C}_{5}$ Capacidade & I & NA & NA \\
\hline
\end{tabular}

Fonte: Elaborado pelos autores.

Aplicando-se a Equação 2 para o cálculo do grau de sobreclassificação das alternativas e em seguida com as Equações 3 e 4, obtém-se os fluxos de saída e entrada do método PROMÉTHÉE II. Finalmente, a diferença entre os dois fluxos fornece a classificação por ordem crescente de fluxos, obtendo assim a classificação final do método. A Tabela 6 mostra um comparativo entre a priorização real definida no portfólio de produtividade elaborado pelos gestores, através de julgamento subjetivo, e o resultante de uma metodologia multicritério. Nota-se que o somatório do fluxo líquido das alternativas do PROMÉTHÉE II deve ser igual a zero.

Tabela 6: Comparativo da priorização PROMÉTHÉE II.

\begin{tabular}{|c|c|c|c|}
\hline \multirow{2}{*}{} & \multicolumn{3}{|c|}{ Priorização } \\
\cline { 2 - 4 } & \multicolumn{2}{|c|}{ PROMÉTHÉE II } & Portfólio \\
\hline Classificação & Projeto & $\Phi$ & Atual \\
\hline $1^{\mathbf{o}}$ & $\mathrm{A}$ & 0,52 & $\mathrm{~A}$ \\
\hline $2^{\mathbf{o}}$ & $\mathrm{H}$ & 0,30 & $\mathrm{C}$ \\
\hline $3^{\mathbf{o}}$ & $\mathrm{C}$ & 0,29 & $\mathrm{H}$ \\
\hline $4^{\mathbf{o}}$ & $\mathrm{B}$ & $-0,06$ & $\mathrm{~B}$ \\
\hline $5^{\mathbf{o}}$ & $\mathrm{D}$ & $-0,07$ & $\mathrm{E}$ \\
\hline $6^{\mathbf{0}}$ & $\mathrm{F}$ & $-0,11$ & $\mathrm{G}$ \\
\hline $7^{\mathbf{0}}$ & $\mathrm{J}$ & $-0,14$ & $\mathrm{~F}$ \\
\hline $8^{\mathbf{o}}$ & $\mathrm{I}$ & $-0,17$ & $\mathrm{D}$ \\
\hline $9^{\mathbf{o}}$ & $\mathrm{E}$ & $-0,20$ & $\mathrm{I}$ \\
\hline $10^{\mathbf{o}}$ & $\mathrm{G}$ & $-0,34$ & $\mathrm{~J}$ \\
\hline
\end{tabular}

Fonte: Elaborado pelos autores.

O comparativo entre as duas priorizações, a atual praticada no portfólio de produtividade e aquela apontada pelo método PROMÉTHÉE II mostra um alinhamento entre os quatro primeiros projetos quanto a sua prioridade. Porém, a partir da quinta posição observa-se uma grande variação na priorização de cada projeto, no comparativo. Isto demonstra, que mesmo sem aplicação de um método multicritério, mas por bem conhecer os critérios importantes, os decisores têm a percepção de quais são os projetos prioritários e de maiores impactos para empresa. Todavia, a mesma percepção não ocorre quando os projetos apresentam menores impactos.

Uma análise de sensibilidade é importante neste processo de avaliação e foi aplicada ao modelo, pois os pesos dos critérios podem sofrer variações em função do momento em que a empresa se encontra, segundo apontado pelos próprios decisores. Em cenários onde o volume de vendas se encontra em baixa, o objetivo de redução de custo se torna prioritário e os critérios $\mathrm{C}_{1}$ (ganho financeiro) e $\mathrm{C}_{5}$ (ganho de capacidade de produção) se alternam como critério prioritário. O resultado da análise mostra que o ranking das alternativas não sofre variações consideráveis e mesmo que ocorra uma variação dos pesos, a priorização dos projetos no portfólio não sofre significativa modificação, conforme mostrado na tabela 7 . 
Tabela 7: Análise de sensibilidade da priorização PROMÉTHÉE II.

\begin{tabular}{|c|c|c|c|}
\hline \multirow{2}{*}{} & \multicolumn{3}{|c|}{ Priorização } \\
\cline { 2 - 4 } & \multicolumn{2}{|c|}{ PROMÉTHÉE II } & Portfólio \\
\hline Classificação & Projeto & $\Phi$ & Atual \\
\hline $1^{\mathbf{o}}$ & $\mathrm{A}$ & 0,51 & $\mathrm{~A}$ \\
\hline $2^{\mathbf{o}}$ & $\mathrm{H}$ & 0,29 & $\mathrm{C}$ \\
\hline $3^{\mathbf{o}}$ & $\mathrm{C}$ & 0,25 & $\mathrm{H}$ \\
\hline $4^{\mathbf{o}}$ & $\mathrm{B}$ & $-0,04$ & $\mathrm{~B}$ \\
\hline $5^{\mathbf{o}}$ & $\mathrm{D}$ & $-0,08$ & $\mathrm{E}$ \\
\hline $6^{\mathbf{o}}$ & $\mathrm{J}$ & $-0,11$ & $\mathrm{G}$ \\
\hline $7^{\mathbf{0}}$ & $\mathrm{F}$ & $-0,12$ & $\mathrm{~F}$ \\
\hline $8^{\mathbf{o}}$ & $\mathrm{I}$ & $-0,16$ & $\mathrm{D}$ \\
\hline $9^{\mathbf{o}}$ & $\mathrm{E}$ & $-0,21$ & $\mathrm{I}$ \\
\hline $10^{\mathbf{o}}$ & $\mathrm{G}$ & $-0,32$ & $\mathrm{~J}$ \\
\hline
\end{tabular}

Fonte: Elaborado pelos autores.

A aplicação do PROMÉTHÉE II auxilia os decisores na composição do portfólio de projetos, definindo suas prioridades. Ele permite que cada alternativa de projeto seja avaliada com todas as outras, sob todos os critérios, evitando amenizar eventual viés causado por decisões intuitivas.

\section{Considerações Finais}

O método multicritério de Apoio à Tomada de Decisão é aplicado amplamente em diversas áreas e pesquisas, sendo um instrumento importante para auxiliar os decisores na priorização de projetos em empresas. Nestas avaliações, é possível que fatores tanto quantitativos quanto qualitativos, sejam considerados na implementação de modelos matemáticos.

A priorização dos projetos de melhoria de produtividade no estudo em questão é realizada de forma subjetiva através dos julgamentos de prioridades dos decisores e o processo de análise das alternativas utilizando o PROMÉTHÉE II gerou um resultado que se mostrou satisfatório. As entrevistas com os decisores responsáveis pela priorização dos projetos na empresa é importante para quantificar os preceitos que subjetivamente já utilizam para planejar os projetos a serem realizados.

Através da aplicação de uma metodologia multicritério, foi possível uma avaliação completa dos projetos de produtividade em todos os critérios avaliados, com resultado robusto e consistente mesmo após uma análise de sensibilidade com variação dos pesos dos critérios. $\mathrm{O}$ método torna mais clara a solução de conflitos existentes entre os critérios e permite aumentar a racionalidade presente no processo de decisão.

A implementação do método PROMÉTHÉE II mostra ao decisor a ordem de preferência de todos os projetos avaliados. Através deste método multicritério, foi possível uma priorização completa do portfólio de projetos de produtividade, sendo capaz de auxiliar na definição de prioridades, seja de projetos com maiores ou menores impactos nos resultados da empresa. $\mathrm{O}$ método PROMÉTHÉE II auxilia os profissionais na construção do plano anual de produtividade, pois permite mapear as preferências dos decisores que conhecem as múltiplas dimensões do problema de avaliação. Além disso, ele permitir aos decisores inferirem parâmetros na realização de preferência nas comparações par a par entre alternativas.

Este processo de avaliação também poderá ser otimizado de acordo com a sua utilização. Novos critérios podem ser acrescentados, assim como uma análise mais completa integrando as restrições de recursos humanos para realização dos projetos, associando o modelo aqui apresentado a outro de mesma família, o PROMÉTHÉE V. 


\section{Referências}

Almeida, J. A. Modelo multicritério para seleção de portfólio de projetos de sistema de informação. 115 f. Tese (Doutorado em Engenharia de Produção) - Universidade Federal de Pernambuco, Recife-PE, 2012.

Almeida, A. T. Processo de Decisão nas Organizações: Construindo modelos de decisão multicritério. São Paulo: Atlas, 2013. 256p.

Almeida, A. T. e Duarte, M. D. O. A multi-criteria decision model for selecting project portfolio with consideration being given to a new concept for synergies. Pesquisa Operacional, v. 31, n. 2, p. 301-318, 2011.

Andersson, C. e Bellgran, M. On the complexity of using performance measures: Enhancing sustained production improvement capability by combining OEE and productivity. Journal of Manufacturing Systems, v. 35, p. 144-154, 2015.

Behzadian, M., Kazemzadeh, R. B., Albadvi, A. e Aghdasi, M. PROMETHEE: A comprehensive literature review on methodologies and applications. European Journal of Operational Research, v. 200, n. 1, p. 198-215, 2010.

Belton, V. e Stewart, T. J. Multiple Criteria Decision Analysis: An integrated approach. Boston: Kluwer Academic Publishers, 2002.

Bin, A., Azevedo, A., Duarte, L., Salles-Filho, S. e Massaguer, P. R\&D and innovation project selection: Can optimization methods be adequate? Procedia Computer Science, v. 55, p. 613$621,2015$.

Brans, J. P. e Mareschal, B. PROMETHEE V: MCDM problems with segmentation constraints. INFOR: Information Systems and Operational Research, v. 30, n. 2, p. 85-96, 1992.

Brans, J. P. e Mareschal, B. The PROMETHEE-GAIA decision support system for multicriteria investigations. ULB Institutional Repository 2013/9367, Universite Libre de Bruxelles, 1994.

Brans, J. P. e Mareschal, B. The PROMETHEE VI procedure: How to differentiate hard from soft multicriteria problems. Journal of Decision Systems, v. 4, n. 3, p. 213-223, 1995.

Brans, J. P. e Mareschal, B. PROMETHEE methods. In: Figueira, J., Greco, S. e Ehrgott, M. (eds.) Multiple Criteria Decision Analysis: State of the Art Surveys, cap. 5, p. 163-186. New York: Springer Science, 2005.

Brans, J. P., Mareschal, B., e Vincke, P. Prométhée: A new family of outranking methods in multicriteria analysis. In: Brans, J. P. (ed.) Operational Research 1984, p. 408-421. NorthHolland, Amsterdam: Elsevier Science Publishers B.V., 1984.

Brans, J. P., Vincke, P. e Mareschal, B. How to select and how to rank projects: The PROMETHEE method. European Journal of Operational Research, v. 24, n. 2, p. 228-238, 1986.

Campos, V. R. Modelo de apoio à decisão multicritério para priorização de projetos em saneamento. Tese (Doutorado em Engenharia de Produção) - Escola de Engenharia de São Carlos, Universidade de São Paulo, São Carlos-SP, 2011. 
Cavalcante, C. A. V. e Almeida, A. T. Modelo multicritério de apoio a decisão para o planejamento de manutenção preventiva utilizando PROMETHEE II em situações de incerteza. Pesquisa Operacional, v. 25, n. 2, p. 279-296, 2005.

CNI - Confederação Nacional da Indústria. Produtividade brasileira é a que menos cresce em relação $\quad a \quad 11$ países. 2015.2 Disponível http://www.portaldaindustria.com.br/agenciacni/noticias/2015/02/produtividade-brasileira-e-aque-menos-cresce-em-relacao-a-11-paises/. Acesso em: 17/06/2017.

Demeter, K., Chikán, A. e Matyusz, Z. Labour productivity change: Drivers, business impact and macroeconomic moderators. International Journal of Production Economics, v. 131, n. 1, p. 215-223, 2011.

Dennis, P. Produção Lean Simplificada: Um guia para entender o sistema de produção mais poderoso do mundo. Porto Alegre: Bookman, 2008.

Ferreira, R. G. Priorização de portfólio de projetos de geração de energia renovável utilizando $o$ método PROMÉTHÉE V. Dissertação (Mestrado Profissionalizante em Administração) Faculdade de Economia e Finanças Ibmec, Rio de Janeiro-RJ, 2013.

Figueiredo, C. J. e Silva, M. H. L. Abordagem multicriterio e lógica fuzzy para priorização de portfólio de produtos em um sistema agroindustrial. Revista Eletrônica Pesquisa Operacional para o Desenvolvimento, v. 6, n. 2, p. 226-242, 2014.

Fontana, M. E. e Morais, D. C. Water distribution network segmentation based on group multicriteria decision approach. Production, v. 27, 2017. http://dx.doi.org/10.1590/01036513.208316.

Gomes, L. F. A. M. Modelagem do risco no apoio à tomada de decisão. Revista de Ciência, Tecnologia e Inovação, v. 1, n. 1, p. 3-14, 2016.

Gomes, L. F. A. M, Gomes, C. F. S. e Almeida, A. T. Tomada de Decisão Gerencial: enfoque multicritério. $3^{\text {a }}$ ed. São Paulo: Atlas, 2009.

Govindan, K. e Jepsen, M. B. ELECTRE: A comprehensive literature review on methodologies and applications. European Journal of Operational Research, v. 250, n. 1, p. 1-29, 2016.

Kaiser, M. G., Arbi, F. E. e Ahlemann, F. Successful project portfolio management beyond project selection techniques: Understanding the role of structural alignment. International Journal of Project Management, v. 33, n. 1, p. 126-139, 2015.

Käpylä, J., Jääskeläinen, A. e Lönnqvist, A. Identifying future challenges for productivity research: evidence from Finland. International Journal of Productivity and Performance Management, v. 59, n. 7, p. 607-623, 2010.

Keelling, R. Gestão de Projetos: uma abordagem global. São Paulo: Saraiva, 2006. 293 p.

Kerzner, H. Gestão de Projetos: as melhores práticas. São Paulo: Bookman, 2006.

King, N. C. O., Lima, E. P. e Costa, S.E.G. Produtividade sistêmica: conceitos e aplicações. Production, v. 24, n. 1, p. 160-176, 2014. 
Kleinmuntz, D. N. Resource Allocation Decisions. In: Edwards, W., Miles, R. F. e von Winterfeldt, D. (eds.) Advances in Decision Analysis: from foundations to applications, cap. 20. Cambridge University Press, 2007.

López, H. M. L. e Almeida, A. T. Utilizando PROMETHEE V para seleção de portfólio de projetos de uma empresa de energia elétrica. Production, v. 24, n. 3, p. 559-571, 2014.

Macedo, M. G. C. Indicadores de sustentabilidade ambiental na indústria da mineração: avaliação pelo método Prométhée II. Dissertação (Mestrado em Administração) - Faculdade de Economia e Finanças Ibmec, Rio de Janeiro-RJ, 2008.

Macedo, M. M. Gestão da produtividade nas empresas. Revista Organização Sistêmica, v. 1, n. 1, p. 110-119, 2012.

Mercado, C. N. e Cleto, M. G. O valor agregado no balanceamento de linha de montagem com abordagem da teoria das restrições: estudo de caso em multinacional no Brasil. Universidade Federal do Paraná, Curitiba-PR, 2012.

Meskendahl, S. The influence of business strategy on project portfolio management and its success - A conceptual framework. International Journal of Project Management, v. 28, n. 8, p. 807-817, 2010.

Montibeller, G. e Franco, A. Decision and risk analysis for the evaluation of strategic options. In: O’Brien, F. A. e Dyson, R. G. (eds.) Supporting Strategy: frameworks, methods and models, p. 251-284. West Sussex: John Wiley \& Sons Ltd, 2007.

Pereira, C. L. C. Modelagem multicriterio para escolha de projetos em fundos setoriais: abordagem aplicada ao fundo setorial audiovisual. Dissertação (Mestrado em Sistemas e Gestão) - Universidade Federal Fluminense, Niterói-RJ, 2016.

PMI - Project Management Institute. Um Guia do Conjunto de Conhecimentos em Gerenciamento de Projetos (Guia PMBOK), $5^{\text {a }}$ ed., 2013.

Rossoni, C. F. Decisão Multicritério, uma pesquisa experimental para avaliação da percepção dos gestores de MPE acerca de um modelo de tomada de decisão multicritério T-ODA quanto à sua aplicabilidade. Dissertação (Mestrado em Administração) - Faculdade Campo Limpo Paulista, Campo Limpo Paulista-SP, 2011.

Roy, B. Classement et choix en présence de points de vue multiple: La méthode Electre. Revue Française D'Informatique et de Recherche Opérationnelle, v. 2, n. 8, p. 57-75, 1968.

Saaty, T. L. The Analytic Hierarchy Process: planning, priority setting, resources allocation. New York: McGraw-Hill, 1980.

Severiano Filho, C. Produtividade \& Manufatura Avançada. João Pessoa: Editora Universitária UFPB, 1998.

Shakhsi-Niaei, M., Torabi, S. A. e Iranmanesh, S. H. A comprehensive framework for project selection problem under uncertainty and real-world constraints. Computers \& Industrial Engineering, v. 61, n. 1, p. 226-237, 2011.

Shimizu, T. Decisão nas Organizações, 2ªd. São Paulo: Atlas, 2006. 
Silva, A. C. G. C., Fontes, C. H. O. e Barbosa, A. S. Multicriteria evaluation model for organizational performance management applied to the Polo Fruit Exporter of the São Francisco Valley. Computers and Electronics in Agriculture, v. 117, p. 168-176, 2015.

Sink, D. S. Productivity Management: Planning, measurement and evaluation, control and improvement. Somerset, New Jersey: John Wiley \& Sons, 1985.

Vegunta, S. C. e Milanovic, J. V. Estimation of cost of downtime of industrial process due to voltage sags. IEEE Transactions on Power Delivery, v. 26, n. 2, p. 576-587, 2011.

Vincke, P. Multicriteria Decision-aid. John Wiley \& Sons, 1992. 